\title{
Antitumor effects of Endostar(rh-endostatin) combined with gemcitabine in different administration sequences to treat Lewis lung carcinoma
}

Cancer Management and Research

\author{
Yuan $\mathrm{Li}^{1}{ }^{1} *$ \\ Pan Huang ${ }^{2, *}$ \\ Hongju Peng' \\ Hongcheng Yue' \\ Min $\mathrm{Wu}^{\prime}$ \\ Shanshan Liu' \\ Rongsheng Qin' \\ Juan Fan' \\ Yunwei Han' \\ 'The Oncology Department, Affiliated \\ Hospital of Southwest Medical University, \\ Lu Zhou, Si Chuan 646000, People's \\ Republic of China; ${ }^{2}$ Neurology \\ Department, Deyang People's Hospital, \\ Deyang, Sichuan 618000, People's \\ Republic of China \\ *These authors contributed equally to \\ this work
}

Background: Endostatin therapy is known to efficiently inhibit angiogenesis and growth of endothelial cells. Nonetheless, the antitumor mechanisms of endostatin combined with chemotherapy remain to be elucidated.

Methods: In our study, a Lewis lung carcinoma transplant mouse model was established and treated with the recombinant human [rh]-endostatin, Endostar, combined with gemcitabine at different sequences. 18F-FDG PET/CT imaging was performed to monitor tumor growth, and hypoxia was examined using an oxygen microelectrode. Vascular endothelial growth factor (VEGF) and alpha smooth muscle actin ( $\alpha$-SMA) levels were detected via immunohistochemistry analysis and cell cycle distributions were analyzed by flow cytometry.

Results: Endostar decreased VEGF expression, improved hypoxia, and influenced cell cycle distributions. Simultaneous treatment of Endostar and gemcitabine displayed significantly tumor inhibition, possessed the lowest uptake of FDG, improved oxygen partial pressure, decreased expression of VEGF, and increased pericyte coverage. Cell cycle analysis demonstrated that cells accumulated in the S phase following gemcitabine treatment and G0/G1 arrest occurred following Endostar treatment. An increase of cells in G0/G1 phase was observed following treatment with Endostar and gemcitabine.

Conclusions: Our study suggests that the combination therapy of Endostar with gemcitabine simutaneously may optimally enhance their individual antitumor effects.

Keywords: recombinant human endostatin, gemcitabine, antiangiogenic, combination therapy

\section{Introduction}

Lung cancer is a major cause for morbidity and mortality worldwide. ${ }^{1}$ Non-small cell lung cancer (NSCLC) constitutes $80 \%$ of lung cancer cases in China, and chemotherapy plays a major role in the treatment of advanced NSCLC. ${ }^{2,3}$ Gemcitabine (GEM) is a cell cycle specific anticancer agent and is administered along with platinum to treat NSCLC. ${ }^{4}$ However, the therapeutic effect of gemcitabine is limited due to strong side effects and development of drug resistance.

The growth and metastasis of tumors depend on neovascularization, which is controlled by both angiogenic and anti-angiogenic factors. Particularly, when solid tumors reach a diameter beyond $2 \mathrm{~mm}$, cancer cells begin to secrete pro-angiogenic factors, which in turn secure tumor growth and development. Currently, several
Correspondence: Juan Fan; Yunwei Han The Oncology Department, Affiliated Hospital of Southwest Medical University, 25 Tai Ping Street, Lu Zhou, Si Chuan 646000,

People's Republic of China

$\mathrm{Tel}+8618683072810$

+86I5883000220

Email fj-joan@I63.com;

530018842@qq.com 
molecules are known to promote angiogenesis in tumors, including vascular endothelial growth factor-A (VEGF-A), placental growth factor (PGF), and basic fibroblast growth factor (bFGF), and the new blood vessels supply oxygen and nutrients to the tumor. ${ }^{5-7}$ Tumor growth, therefore, can be achieved by blocking these angiogenic molecules. Thus, angiogenic inhibitors have served as successful cancer treatments in subsets of cancer patients, and more clinical trials are on the way to increase the repertoire of angiogenic inhibitors available for cancer treatment.

Endostatin is a $20-\mathrm{KDa} \mathrm{C}$-terminal fragment originated from Collagen XVIII, and has the broadest anti-cancer spectrum among endogenous angiogenesis inhibitors. Importantly, more than $12 \%$ of the angiogenic regulatory genes found in the human genome are regulated by endostatin. ${ }^{89}$ Although the molecular mechanisms regulating endostatins remain unclear, various line of research suggest that endostatins can block cell responses to angiogenesis molecules, such as VEGF and FGF, which in turn inhibits vascular endothelial proliferation and migration. ${ }^{10,11}$ Given that endostatins have been shown to lack efficacy, a novel human recombinant (rh) version of endostatin that is expressed in Escherichia coli called Endostar (rhendostatin) was approved by the United States Federal Drug Administration (FDA) in 2005 for treatment of NSCLC. ${ }^{12}$ Notably, compared with endostatin, the stability and biological activity of Endostar was greatly enhanced.

The structure and function of the tumor vasculature is abnormal in nature, resulting in a hypoxic tumor microenvironment with high permeability. ${ }^{13,14}$ Despite the traditional views on antiangiogenesis, Jain et al found that antiangiogenic therapy can decrease tumor blood vessel density and augment pericyte coverage, inducing tumor vasculature structure to normalize vascular function. Additionally, these vasculature changes can reduce tumor blood vessel leakiness, thereby improving the tumor's hypoxic environment. During this tumor developmental period, anticancer treatments can be more effective when used in combination with radiotherapy or chemotherapy modalities. Previous studies have suggested that endostatin alone is not effective in suppressing tumor growth and metastasis unless combined with chemotherapy. ${ }^{15-18}$ For instance, Zhu et al found that esophageal squamous carcinoma could be inhibited more effectively when Endostar was used in combination with radiotherapy. ${ }^{19}$ Moreover, Ren et al showed that Endostar had an additive effect with cyclophosphamide (CTX) and cis-

diamminodichloroplatinum (DDP) in tumors derived from the B16-F10 melanoma cell line or the A549 NSCLC cell line. ${ }^{20}$ Nevertheless, the process of tumor vasculature normalization is transient and due to the complexity underlying the mechanisms regulating angiogenesis, the most effective administration schedule for the combined treatment using endostar and chemotherapy is unclear; therefore, the best administration schedule of endostar is important for improving treatment regimes for NSCLC. The present study worked towards defining an optimal combination treatment using Endostar in combination with gemcitabine in a murine Lewis lung carcinoma (LLC) xenograft cancer model.

\section{Materials and methods \\ Mice, reagents, and cell lines}

C57BL/6 female mice, 6-8 weeks old, were purchased from Tengxin Biotechnology Co. (Chongqing, China) and housed at $25-29{ }^{\circ} \mathrm{C}, 50-76 \%$ humidity at a $12 / 12$ light/dark cycle. All experiments involving animals were reviewed and approved by the Institutional Animal Care and Treatment Committee of the Southwest Medical University, Sichuan, China. And all animal experiments were performed according to the National Institutes of Health guide for the care and use of Laboratory animals (NIH Publications No. 8023, revised 1978).

LLC cell line used in our experiment were purchased by the Experimental Research Center of the Affiliated Hospital of Southwest Medical University (Luzhou, China) from the Cell Bank of the Chinese Academy of Sciences and maintained in PPMI-1640 cell culture media. About $1 \times 10^{6}$ of LLC cells in $0.1 \mathrm{ml}$ of phosphate-buffered saline (PBS) were injected subcutaneously (SC) into the right proximal hind leg of C57BL/6 mice.

The human recombinant endostatin, Endostar (rh-ES), was kindly provided by Shandong Simcere Medgenn Biopharmaceutical Co. (Yantai, Shandong, China) and stored at $4{ }^{\circ} \mathrm{C}$. Endostar was then dissolved in $0.2 \mathrm{ml}$ of $0.9 \%$ normal saline (NS) and administrated to each animal at a dose of $10 \mathrm{mg} / \mathrm{kg} /$ day by intraperitoneal (IP) injection.

Gemcitabine was purchased from Haosen Pharmaceutical Co. (Jiangsu, China). According to previous studies, ${ }^{21}$ gemcitabine was dissolved in $0.2 \mathrm{ml} 0.9 \% \mathrm{NS}$ and administrated to each animal at a dose of $30 \mathrm{mg} / \mathrm{kg}$ by IP injection.

\section{Treatment groups}

10 days after LLC inoculation, tumor-bearing mice were randomly divided into control group, Endostar (ES) alone, gemcitabine (GEM) alone, Endostar first (ES/GEM), 
gemcitabine first (GEM/ES), Endostar and gemcitabine simultaneously (ES + GEM) group ( $\mathrm{n}=12$, per group) [Figure 1]. In brief, i) control group (negative control) was left untreated; ii) ES alone (10 mg/kg/d for 14 days); iii) GEM alone (30 mg/kg/day administered on day 1 and 8 ); iv) ES/GEM (Endostar administered on days 1-14, and gemcitabine administered on days 5 and 12); v) GEM/ES (Endostar administered on days 2-15, and gemcitabine administered on days 1 and 8); vi) ES + GEM (Endostar administered on days $1-14$, and gemcitabine administered on days 1 and 8 ). Tumor volume was measured every two days by caliper and the tumor volume was calculated according to the following formula: $\mathrm{V}=1 / 2 \mathrm{ab}^{2}$ (where $\mathrm{V}$ is the tumor volume, $a$ is the length of the major axis, and $b$ is the length of the minor axis). The mice were sacrificed after the treatment ended.

\section{Flow cytometry analysis}

Tumor tissue was dissected and prepared into a single cell suspension. The single cell suspension was fixed in $70 \%$ cold ethanol for 12-24 hrs. Next, the suspension was precipitated by centrifugation at $1,000 \mathrm{r} / \mathrm{min}$ for $1 \mathrm{~min}$. The cell was rinsed once in PBS ( $\mathrm{pH}$ 7.4) for 2-3 mins and centrifuged at 2,000 r/min for 3-4 mins. Then the sample was stained with propidium iodide (PI) for cell cycle analysis.

\section{Immunohistochemistry}

Tumor tissues were fixed in 10\% neutral formaldehyde, embedded in paraffin and cut into $3 \mu \mathrm{m}$ sections. Then, the sections were stained with the hematoxylin and eosin (H\&E) dye. Subsequently, the sections were treated with specific antibodies for analysis. Briefly, the sections were stained with rabbit anti-mouse VEGF or alpha-smooth muscle actin ( $\alpha$-SMA) primary antibodies and incubated with horseradish peroxidase (HRP)-conjugated goat antimouse secondary antibodies. Next, the slides were visualized by incubation with diaminobenzidine (DAB) and counter-stained with hematoxylin.

For VEGF expression analysis, we calculated as follows: five areas were selected randomly from each of the slides generated from the 6 tumors obtained per group. The number of VEGF positive cells were divided by the total cell count $\times 100 \%$ under $400 \times$ magnification. A medium or intense brown cytoplasmic staining was deemed as positive, while cells with unstained or light brown cytoplasmic staining were deemed as negative.

For $\alpha$-SMA expression analysis, we calculated as follows: five areas were selected randomly from each of the slides generated from the 6 tumors obtained per group. The number of $\alpha$-SMA positive profiles per $\mathrm{mm}^{2}$ of tumor sample was calculated under a $200 \times$ magnification, as previously described. ${ }^{22}$

\section{Oxygen microelectrode}

The partial pressure of oxygen $\left(\mathrm{PO}_{2}\right)$ in tumor tissue was determined by an oxygen microelectrode (Unisense $\mathrm{A} / \mathrm{S}$, Aarhus, Denmark). All operations were followed as described in the manufacturer's instructions. Briefly, after the microelectrode was polarized and calibrated, anesthetized animals were placed in the prone position. Next, the microelectrode was insert into the tumor tissue vertically. The $\mathrm{PO}_{2}$ located at $1 / 2$, $1 / 4,1 / 8$, and $1 / 16$ short diameter of tumor tissue was calculated. The data obtained were then recorded using the dataacquisition software, SenseTrace Pro (Unisense A/S). In order to observe a variation in hypoxia, the $\mathrm{PO}_{2}$ of five animals from each group on the 5th, 10th, and 15th was measured.

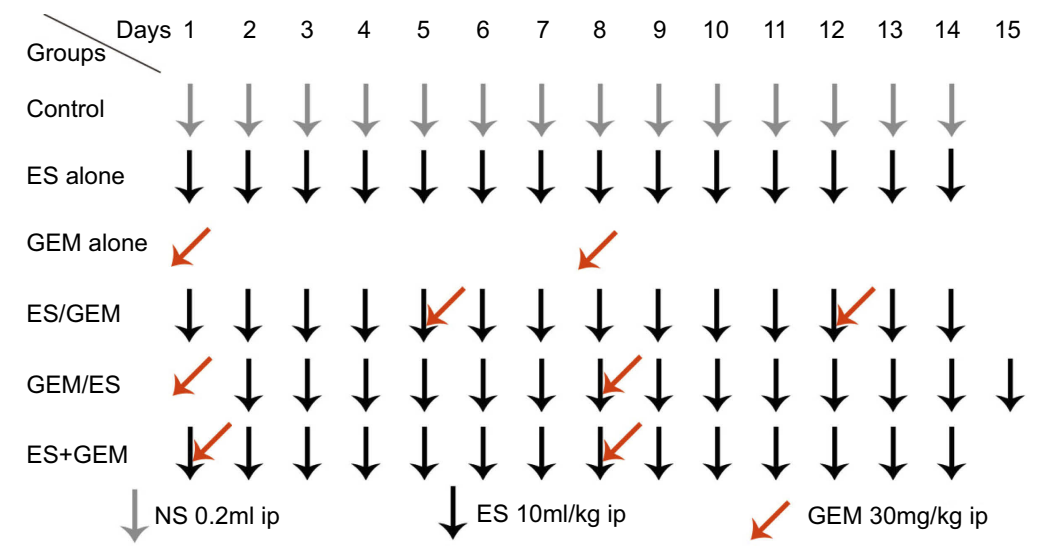

Figure I Treatment schedule.

Abbreviations: Ip, intraperitoneal; ES, endostar; GEM, gemcitabine. 


\section{Micro I8F-FDG PET/CT imaging}

The early tumor response in different groups was measured by an Inveon micro PET/CT animal scanner (Siemens, Munich, Germany). After the day of treatment ended, 3 mice from each group were fasted for 6-8 hrs, then anesthetized with $1 \%$ pentobarbital ( $5 \mathrm{mg} / \mathrm{kg}$, IP) and injected intravenously (IV) with 2-deoxy-2-[fluorine-18] fluoro-D-glucose $\left({ }^{18} \mathrm{~F}-\mathrm{FDG}\right)$ 100-200 micro curie ( $\mu \mathrm{Ci}$ ). After $30 \mathrm{mins}$ of ${ }^{18}$ F-FDG injection administration, treated mice were then fixed to the scanning frame in order to perform micro positron emission tomography integrated with computed tomography (PET/CT) imaging. The image plane of the largest tumor appearance was selected for data analysis. An irregular region of interest (ROI) containing the whole tumor was drawn manually. According to the ROI, the tracer uptake value in the tumor was defined in the attenuation-corrected transaxial tomographic slice by computing the standard uptake value. The maximal standardized uptake value (SUVmax) acquired from the selected ROI.

\section{Statistical analyses}

All data were expressed as the mean \pm standard deviation (SD) and analyzed using the SPSS version 17.0 (IBM, Chicago, IL, USA). Statistical analysis was performed using the one-way ANOVA test. The StudentNewman-Keuls (SNK) method was used as a post-hoc test. $P$-value less than $(<) 0.05$ was considered to be statistically significant.

\section{Results}

\section{Tumor volume}

In our study, we evaluated the antitumor effects of Endostar in combination with gemcitabine on the growth of LLC tumors (Figure 2A) and we determined their optimal administration sequence in vivo using a mouse model. Drug treatments were initiated when the tumor volume diameter reached approximately $8-10 \mathrm{~mm}$. At the end of the treatment (Figure 2A and $\mathrm{C}$ and Table 1), the tumors from the combination therapy, including GEM/ES $\left(2284.83 \mathrm{~mm}^{3}\right)$ and ES/GEM $\left(2882.15 \mathrm{~mm}^{3}\right)$, were significant smaller than ES alone $\left(4386.95 \mathrm{~mm}^{3}, P<0.05\right)$ or GEM alone $\left(3452.79 \mathrm{~mm}^{3}\right.$, $P<0.05)$. The tumor volume was lowest in the ES + GEM group $\left(1758.67 \mathrm{~mm}^{3}\right)$ compared to all other treatment groups $(P<0.05)$, whereas GEM alone group only modestly suppressed tumor growth compared to the control (4767.43 $\left.\mathrm{mm}^{3}, P<0.05\right)$. Consistent with the changes in tumor volume, the tumor weight (Figure $2 \mathrm{~B}$ and Table 1) of the ES + GEM group $(1.91 \mathrm{~g})$ was significant lighter than the control (5.82 g, $P<0.05$ ), ES alone (5.14 g, $P<0.05)$, GEM alone (4.37 g, $P<0.05)$, ES/GEM (3.46 g, $P<0.05)$, and GEM/ ES (3.12 g, $P<0.05)$.

\section{Tumor oxygenation}

Angiogenesis can induce tumor vasculature tends to be normal, thus hypoxia of tumor is improved. In our study, tumor oxygenation was calculated using an oxygen microelectrode on day 5, 10, and 15 of treatment (Figure 3). As shown in Figure 3, the oxygenation levels of the ES alone group was significantly higher on day $5(27.06 \mathrm{mmHg})$ and day $10(23.46 \mathrm{mmHg})$ compared with the control
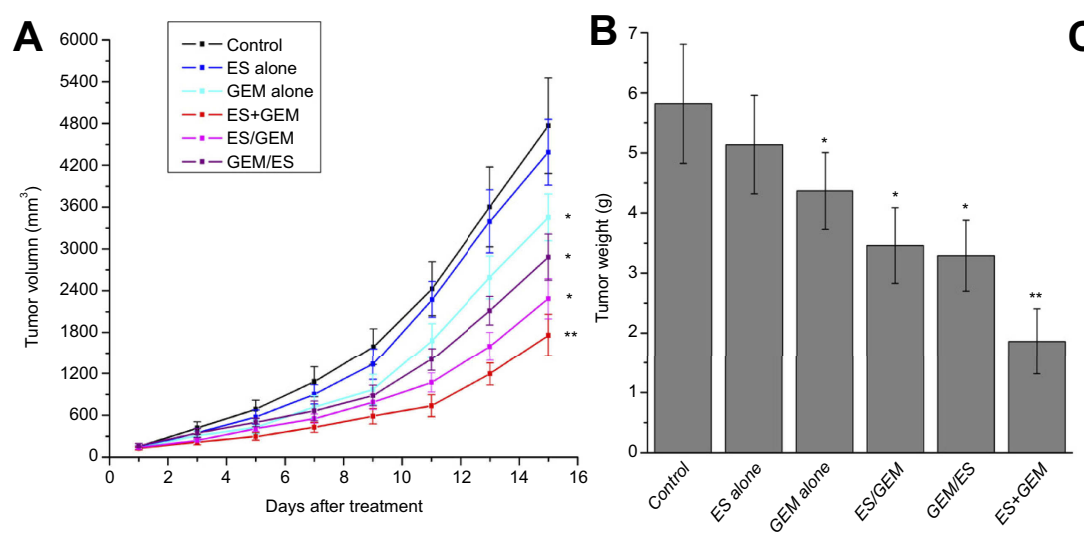

C

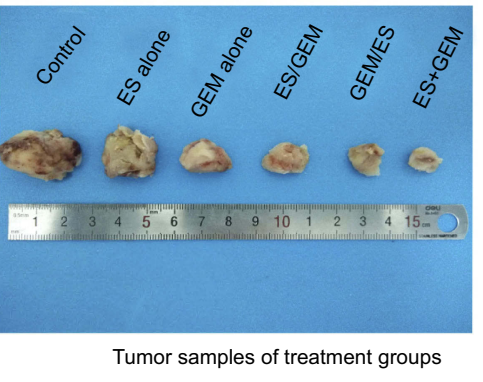

Figure 2 Tumor size, tumor weight and tumor samples of various treatment regimens. (A) Suppression of tumor growth after different treatment regimens in C57/BL6 mice xenografted with LLC. (B) Mean tumor weights of mice from different treatment regimens. (C) Tumor samples of mice from different treatment regimens. $* P<0.05$ vs control group, $* * P<0.05$ vs all groups.

Abbreviations: Ip, intraperitoneal; ES, endostar; GEM, gemcitabine; LLC, Lewis lung carcinoma. 
Table I Tumor volume and tumor weight in LLC xenografted mice with various treatment regimens at the end of treatment

\begin{tabular}{|l|l|l|}
\hline Groups & Tumor volume $\left(\mathbf{m m}^{\mathbf{3}}\right)$ & Tumor weight $\mathbf{( g )}$ \\
\hline Control & $4767.43 \pm 986.57$ & $5.82 \pm 0.99$ \\
ES alone & $4386.95 \pm 732.88$ & $5.14 \pm 0.82$ \\
GEM alone & $3452.79 \pm 434.72$ & $4.37 \pm 0.64$ \\
ES/GEM & $2882.15 \pm 414.2$ & $3.46 \pm 0.63$ \\
GEM/ES & $2284.83 \pm 391.29$ & $3.13 \pm 0.62$ \\
ES + GEM & $1758.67 \pm 426.92$ & $1.91 \pm 0.47$ \\
\hline
\end{tabular}

Abbreviations: LLC, Lewis lung carcinoma; ES, Endostar; GEM, gemcitabine.

group (18.26 mmHg, $15.58 \mathrm{mmHg}, P<0.05$ ), while no obvious differences were observed between the ES alone
(15.40 $\mathrm{mmHg}$ ) and the control group (11.55 $\mathrm{mmHg}$ ) on day 15 of treatment $(P>0.05)$. Compared with all other groups, PO2 was highest in the ES + GEM group on day $5(37.33 \mathrm{mmHg})$, day $10(33.81 \mathrm{mmHg})$, and day $15(27.35 \mathrm{mmHg})$ of treatment $(P<0.05)$.

\section{Micro I8F-FDG PET/CT imaging}

The SUVmax values of $18 \mathrm{~F}-\mathrm{FDG}$ imaging for each group were (Figure 4): $4.2 \pm 0.4$ for control, $3.83 \pm 0.26$ for $\mathrm{ES}$ alone, $3.4 \pm 0.2$ for GEM alone, $2.9 \pm 0.21$ for ES/GEM, $2.3 \pm 0.21$ for $\mathrm{GEM} / \mathrm{ES}$, and $1.73 \pm 0.12$ for $\mathrm{ES}+\mathrm{GEM}$. The SUVmax values in the GEM alone group decreased

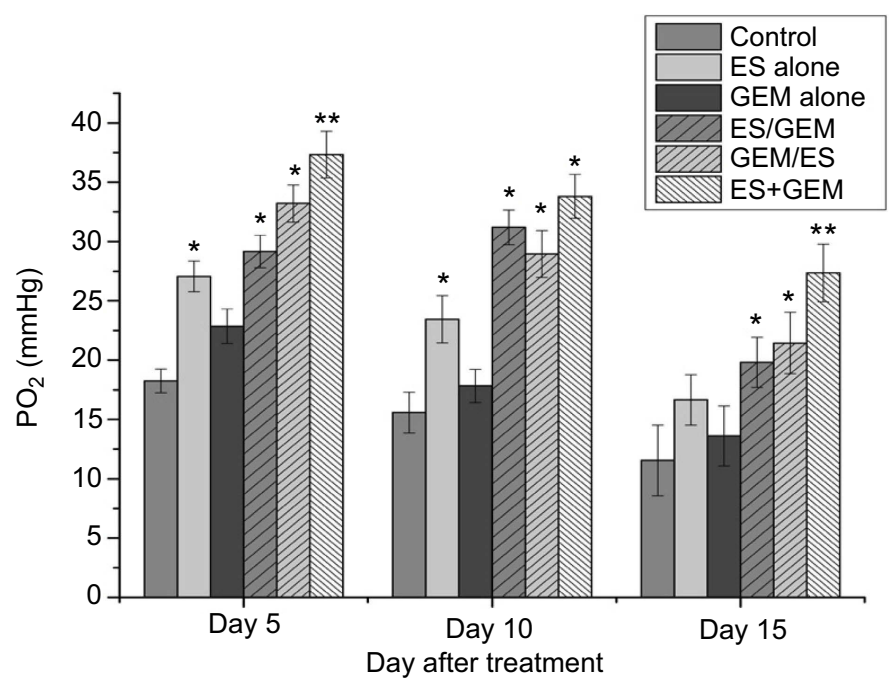

Figure 3 Tumor oxygenation. The oxygenation levels in different groups on day 5 , day 10 , dayl 5 after treatment. Results are expressed as mean \pm SD. $* P<0.05$ vs control group, $* * P<0.05$ vs all groups.
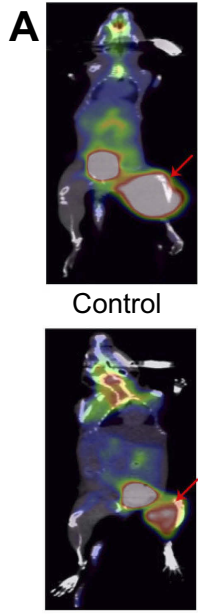

ES/GEM

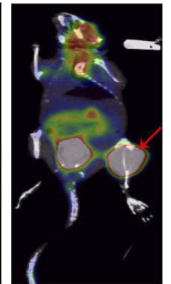

ES alone

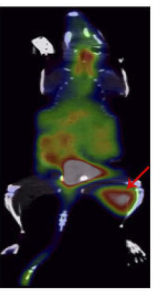

GEM/ES
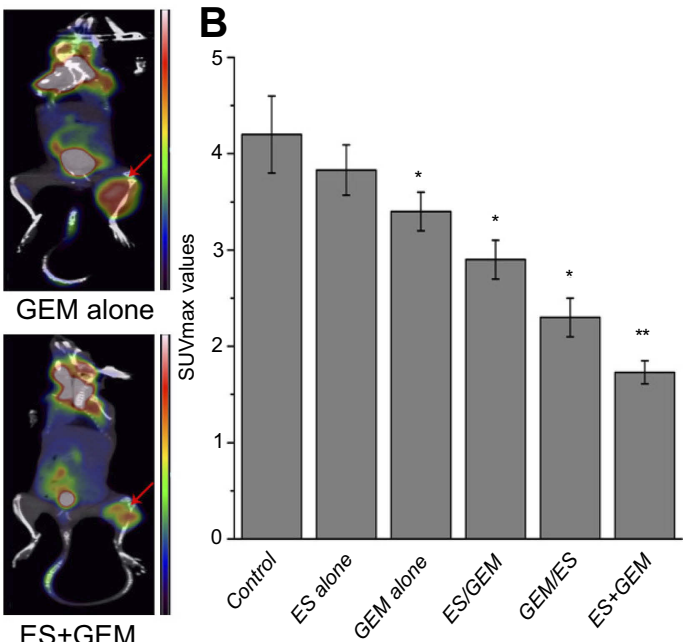

Figure 4 Micro I8F-FDG PET/CT imaging. (A) Representative I8F-FDG PET scans of mice one day after treatment with various regimens. (B) SUVmax associated with various treatment regimens. $* P<0.05$ vs control group, $* * P<0.05$ vs all groups.

Abbreviations: ES, endostar; GEM, gemcitabine. 
considerably compared with the control $(P<0.05)$. Among all treatment groups, tumor cells in the ES + GEM group had the lowest SUVmax value $(P<0.05)$. Surprisingly, no significant differences in SUVmax were observed between the ES alone group and the control group $(P>0.05)$.

\section{Tumor cell cycle analysis}

After treatment completion, the tumor cell cycle was evaluated by flow cytometry in each of the treatment groups (Figure 5). Following the GEM alone treatment, the proportion of cells in the synthesis (S) phase $(26.3 \%)$, increased slightly compared to the control $(21.64 \%, P<0.05)$. Whereas, compared to the control $(35.63 \%)$ and GEM alone group (39.63\%), the cell cycle distribution in the G0/G1 phase, dedicated to cell division preparation, was remarkably higher in the ES alone group $(47.35 \%, P<0.05)$. In particular, the $\mathrm{ES}+\mathrm{GEM}$ group had the highest $\mathrm{G} 0 / \mathrm{G} 1$ phase $(59.16 \%)$ and $\mathrm{S}(39.09 \%)$ phase fraction compared to the control $(P<0.01)$.

\section{Immunohistochemistry}

The expression of VEGF was evaluated via immunohistochemistry in order to determine the impact of Endostar and gemcitabine treatments (Figure 6). Both the control group (91.35\%) and the GEM alone group (87.99\%) had a higher number of VEGF positive cells compared to the ES alone (69.38\%, $P<0.05)$, ES/GEM (51.93\%, $P<0.05)$, GEM/ES (45.38\%, $P<0.05)$, and the ES + GEM (27.18\%, $P<0.05)$.
Tumors cells that were treated with ES + GEM had the lowest VEGF expression observed in all groups $(P<0.05)$.

Moreover, $\alpha$-SMA staining was performed to reveal the extent of angiogenesis taking place after various treatments (Figure 7). Compared with the control group (4.17 vessels $/ \mathrm{mm}^{2}$ ), the number of $\alpha$-SMA positive blood vessels increased significantly in the ES alone (6.83 vessels $\left./ \mathrm{mm}^{2}, P<0.05\right)$, ES/GEM (8.67 vessels $/ \mathrm{mm}^{2}$, $P<0.05)$ and GEM/ES group (8.5 vessels $/ \mathrm{mm}^{2}, P<0.05$ ). However, no obvious differences were found between the GEM alone (4.67 vessels $/ \mathrm{mm}^{2}$ ) and the control group $(P>0.05)$. In addition, the number of $\alpha$-SMA positive blood vessels in the ES + GEM group (10.33 vessels/ $\mathrm{mm}^{2}$ ) was the highest among all the groups $(P<0.05)$.

\section{Discussion}

It is now well accepted that a network of blood vessels develops to supply nutrients and oxygen to facilitate tumor cell growth, invasion, and metastasis. Based on this concept, anti-angiogenic therapy is a crucial approach for efficient antitumor treatments. Consistently, a myriad of studies investigating novel antiangiogenic therapies have erupted in the last years. Nevertheless, several studies have shown that monotherapy treatments geared toward the inhibition of angiogenesis have performed poorly on tumor inhibition. ${ }^{23,24}$ Importantly, the therapeutic efficacy of anti-angiogenic approaches is limited because of their major role in

A

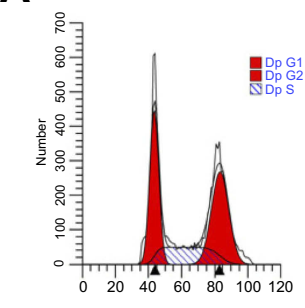

Control

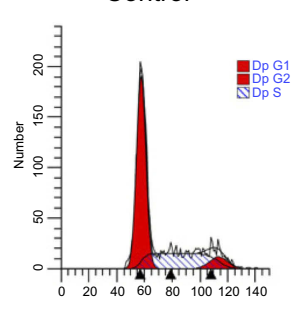

ES/GEM

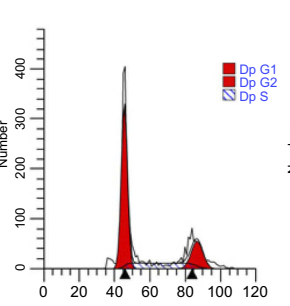

ES alone

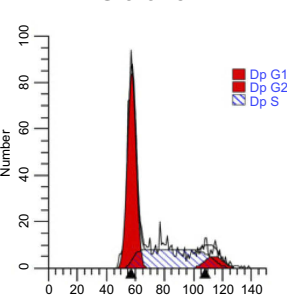

GEM/ES

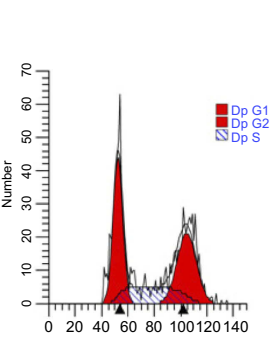

GEM alone

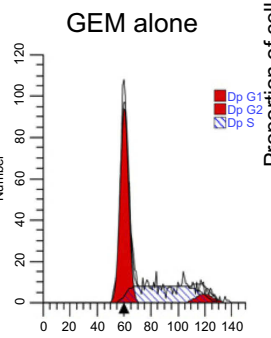

ES+GEM
B

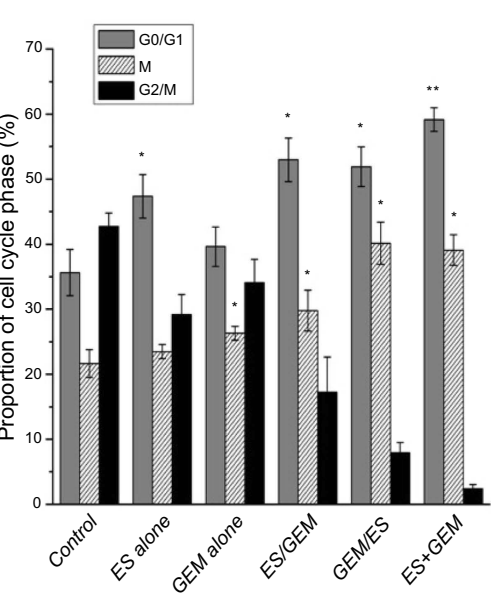

Figure 5 Cell cycle analysis of tumor tissue from mice in different groups. (A) Flow cytometry analysis of cell cycle redistribution in xenografts from mice in different groups. (B) Percentage of cells in G0/GI, S, G2/M phase in mouse xenografts in different treatment groups. $* P<0.05$ vs control group, $* * P<0.05$ vs all groups. Abbreviations: ES, endostar; GEM, gemcitabine. 
A

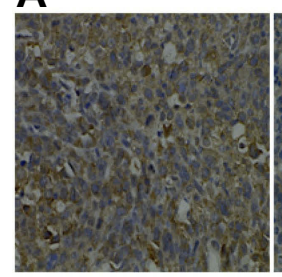

Control

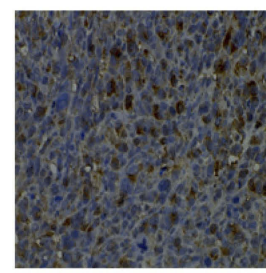

ES/GEM

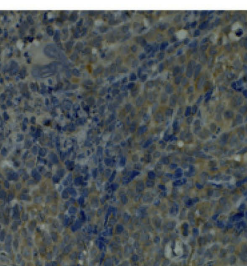

ES alone

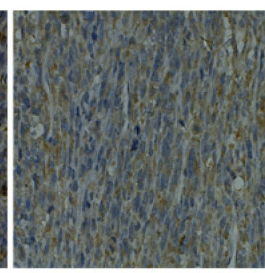

GEM/ES

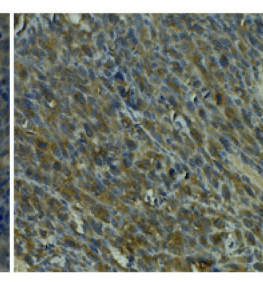

GEM alone

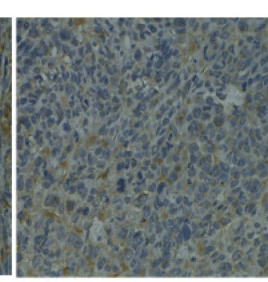

$E S+G E M$

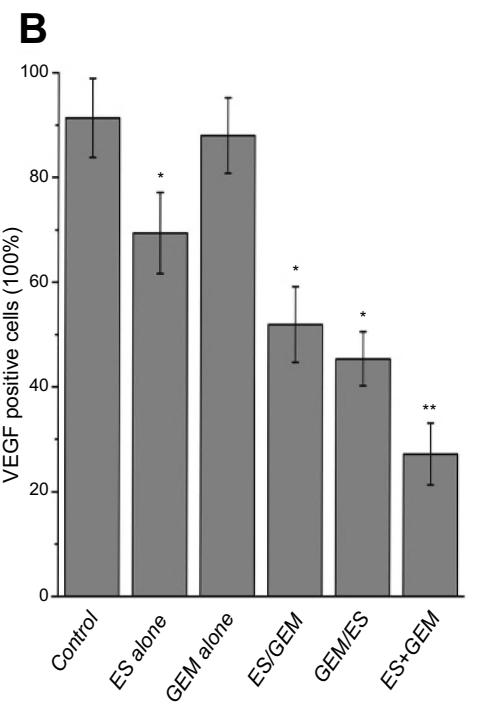

Figure 6 Expression of VEGF in Lewis tumor tissue. (A) Immunohistochemical staining against VEGF of xenografted Lewis treated tissues under various treatment regimens ( $\times 400$ magnification). (B) VEGF positivity stain (\%) within treatment groups. $* P<0.05$ vs control group, $* * P<0.05$ vs all groups.

Abbreviations: ES, endostar; GEM, gemcitabine.

A

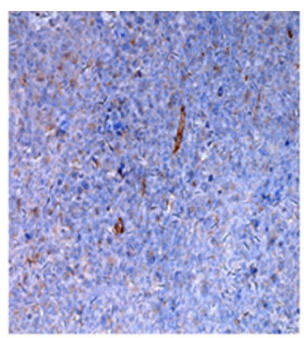

Control

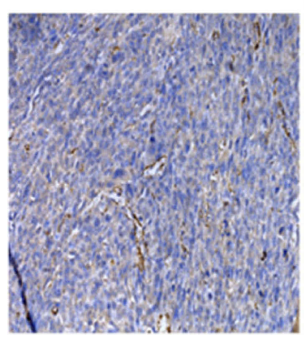

ES/GEM

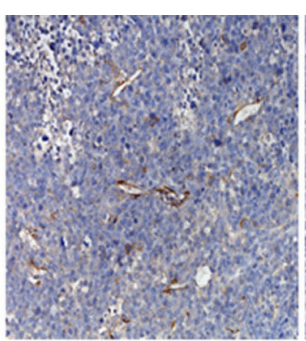

ES alone

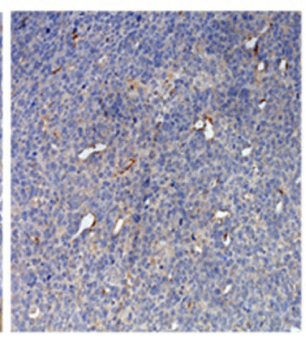

GEM/ES

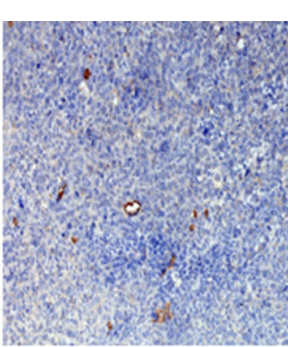

GEM alone

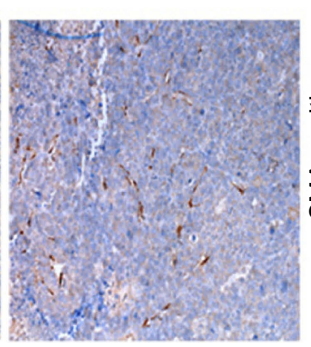

$E S+G E M$

\section{B}

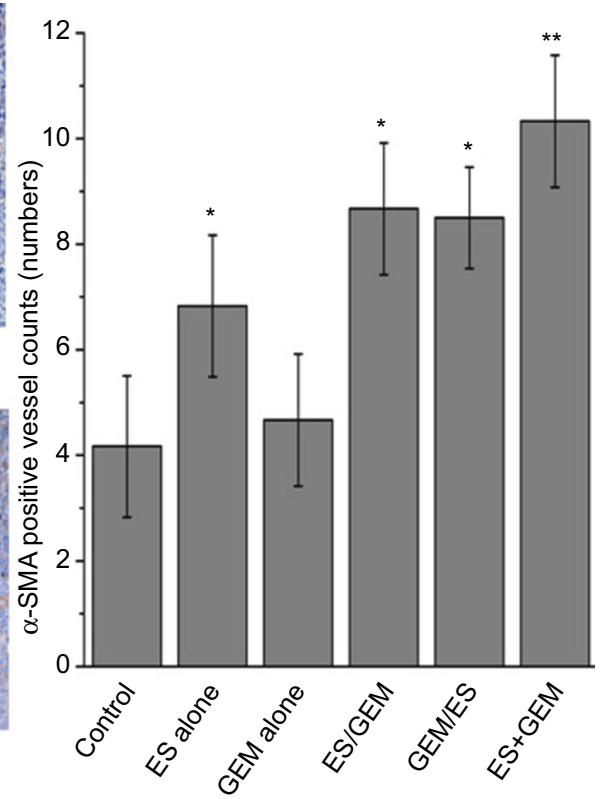

Figure 7 Expression of $\boldsymbol{\alpha}$-SMA in Lewis tumor tissue. (A) Immunohistochemical staining against $\boldsymbol{\alpha}$-SMA in xenografted Lewis treated with various treatment regimens $(\times 200$ magnification). (B) Number of $\alpha$-SMA positive vessels within treatment groups. $* P<0.05$ vs control group, $* * P<0.05$ vs all groups.

Abbreviations: ES, endostar; GEM, gemcitabine.

angiogenesis, as with tumor treatment with the VEGF-A inhibitor, bevacizumab (Avastin, Roche). When tumors possess abnormal vascular networks, tumor cells become less sensitive to chemotherapy. Despite these disadvantages, preclinical and clinical studies indicate that antiangiogenic therapy in combination with chemotherapy is more effective than single anti-angiogenic modalities in cancer treatments. ${ }^{24-28}$ In the present study, our data also confirm that a combination therapy has advantages as antitumor treatment, compared to monotherapies. However, despite these findings, the optimal administration schedule for a combination therapy consisting of 
anti-angiogenic agents and chemotherapy treatments has not been fully elucidated.

In agreement with previous studies, ${ }^{24}$ the present study demonstrates that Endostar monotherapy does not suppress tumor growth effectively, both in terms of tumor volume and tumor weight. In contrast, the combination therapy of Endostar and gemcitabine suppresses tumor growth in an appreciable manner relative to monotherapy treatment with either drug. The positive effect of this treatment was also assessed using 18F-FDG PET/CT imaging. 18F-FDG, an analogue of glucose, is widely used as a functional molecular biomarker for PET imaging, as it allows for the visualization for glucose metabolism. It is now well known that many biological activities require energy, which is essential for proper cell metabolism. Unlike normal cells, the majority of the energy used by tumor cells relies on glycolysis, mediated by glycometabolic pathways, and not by aerobic oxidation. ${ }^{29}$ Thus, inhibiting proliferation can weaken glycometabolism. Importantly, higher FDG uptake reflect rapid glucose metabolism, whereas poor FDG uptake implies a reduction in glucose metabolism. Thus, the use of the radiotracer 18F-FDG PET/CT is an important approach to predict tumor malignancy in a clinical setting. Our data suggest that combination therapy leads to slower FDG uptake compared to Endostar monotherapy, indicating that Endostar combined with chemotherapy treatment can enhance its antitumor effect. In addition, H\&E staining of the heart, liver, spleen, lung, and kidney from each of the treatment groups was evaluated microscopically (Figure 8). Notably, no significant morphological changes in these organs were found, which indicates that the combination therapy used did not have obvious adverse impacts on normal tissues.

This phenomenon of augmented antitumor effect under a combination therapy of Endostar with chemotherapy is associated with changes in tumor vascular normalization. As previously described, antiangiogenic treatments have been shown to result in tumor vascular functional and structural normalization. ${ }^{16,30,31}$ Thus, the normal basement membrane increases, such as pericytes, which enhances drug delivery into tumors. Pericytes as a key characteristic of mature microvessels, such as making contact of endothelial closer and enhancing the stability of vessels. ${ }^{32,33}$ Pericytes were assessed using $\alpha$-SMA, a maker for the maturity and function of blood vessels. The present study demonstrates that the combination of Endostar and gemcitabine result in higher number of pericytes compared to the control or gemcitabine only treatment groups. These results indicate that Endostar treatment led to higher number of mature blood

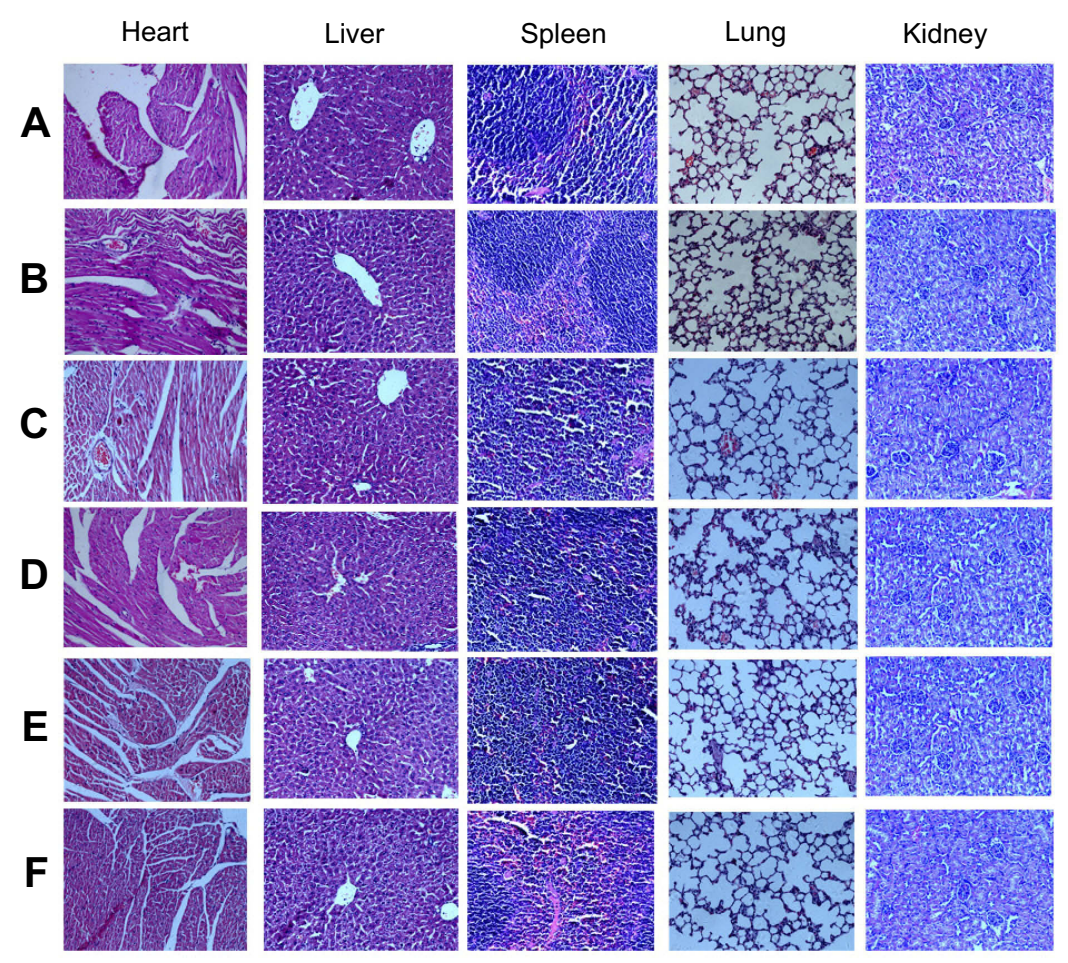

Figure 8 Organs morphology of mice from different groups. Hematoxylin-eosin staining of the heart, liver, spleen, lung, kidney in different groups. (A) Control group, (B) ES alone group, (C) GEM alone group, (D) ES/GEM group, (E) GEM/ES group, and (F) ES + GEM group.

Abbreviations: ES, endostar; GEM, gemcitabine. 
vessels and normalized function of the tumor vasculature. Therefore, the antitumor effects were enhanced compared to a single agent therapy. Hypoxia is common in solid tumors and influences the sensitivity of tumors to radiotherapy and chemotherapy. A previous study found that hypoxia improves after antiangiogenesis therapy. ${ }^{34}$ In our study, Endostar alone and the three combined treatments all induced a higher oxygen partial pressure on days 5 and 10 of the treatment, suggesting that Endostar improved hypoxia and increased the antitumor efficiency of chemotherapy.

Peng et al found that the therapeutic effect improved after radiotherapy treatments were delivered following Endostar administration for 5-7 days in xenografts of the human nasopharyngeal carcinoma (NPC) cell line CNE-2 (CNE-2 NPC) or following Endostar administration for 3-5 days in 5-8F NPC xenografts. ${ }^{35}$ On the contrary, we found that tumor volume was the lowest and had the slowest FDG uptake in the ES + GEM group after 15 days of treatment. These results suggest that administration of Endostar with gemcitabine simultaneously, is the optimal combination therapy, compared to successive administration. In addition, according to Huang et al treatment with the chemotherapy agent paclitaxel followed by Endostar treatment may also induce a synergistic effect when compared to treatment of each drug alone. ${ }^{36}$ However, in our study, the antitumor effect observed in the ES + GEM group was more powerful than the effect seen with the ES/GEM or GEM/ES groups. We hypothesize that this phenomenon may be due to a modulation of the VEGF pathway.

VEGF signals play a vital role in angiogenesis. Several studies have suggested that the expression of VEGF is enhanced after chemotherapy treatments. ${ }^{37,38}$ It has also been reported that chemotherapy drugs may inhibit VEGF expression, and these drugs may also induce angiogenesis. $^{39,40}$ Endostar is an antiangiogenic agent, but its mechanism of action is still not fully understood. Moreover, gemcitabine could inhibit VEGF expression early in treatment but increase VEGF expression with long-term treatment. ${ }^{21}$ In our study, the GEM alone treatment showed no obvious influence on VEGF expression relative to the control group at the end of the treatment. Interestingly, gemcitabine administration with Endostar simultaneously decrease the expression of VEGF significantly. This indicates that Endostar has the potential to downregulate VEGF expression, consistent with previous studies by $\mathrm{Li}$ et $\mathrm{al}^{41}$. Furthermore, VEGF inhibition was enhanced when Endostar was administered with gemcitabine simultaneously.

Gemcitabine, as a cytotoxic drug, acts mainly on tumor cells in the $\mathrm{S}$ phase. ${ }^{42}$ In the present study, we also observed a cell cycle arrest in the $S$ phase with the gemcitabine treatment. Therefore, the cell cycle was blocked and tumor cells were not able to complete proliferation. Interestingly, our study demonstrates that there was more cells in the G0/G1 phase Endostar treatment and that Endostar may influence cell cycle distribution. This finding has also been reported by $\mathrm{Xu}$ and colleagues. ${ }^{43}$ When exposed to a combination of Endostar and gemcitabine simultaneously, the proportions of cells in the G0/G1 and $\mathrm{S}$ phases both increased sharply. Our data suggest that simultaneous treatment of Endostar and gemcitabine regulate the cell cycle in different phases, thereby generating an additive effect. Additionally, it indicates that gemcitabine might also induce cell cycle arrest in the G0/G1 phase under conditions involving the simultaneous administration of these two agents.

In conclusion, our study demonstrates that the simultaneous treatment of Endostar and gemcitabine exerts synergistic effects as antitumor therapy. Nevertheless, our data does not confirm an optimal time window for the treatment of Endostar in combination with gemcitabine. Du et al have reported that a lower dose of Endostar $(10 \mathrm{mg} / \mathrm{kg})$ in combination with cisplatin, administered simultaneously, can have a synergistic effect than each therapy alone, while in a study published by Li et al, an observed synergistic therapeutic effect was seen with a dose of $20 \mathrm{mg} / \mathrm{kg}$ of Endostar followed by cisplatin treatment. $^{41,44}$ Thus, we hypothesize that this time window may be associated with the dosage of Endostar. Further studies are needed to explore the optimal time windows associated with different doses of Endostar alone and in combination with other antitumor agents. In addition, the positive effects of these chemotherapy combinations, as well as the subset of patients that may benefit the most from these treatments.

\section{Acknowledgments}

The author would like to thank the members of the department of Pathology and Nuclear medicine, oncology laboratory of Affiliated Hospital of Southwest Medical University for providing assistance throughout the duration of the study. All authors declare that they have no competing interests. 


\section{Disclosure}

The authors report no conflicts of interest in this work.

\section{References}

1. Bray F, Ferlay J, Soerjomataram I, Siegel RL, Torre LA, Jemal A. Global cancer statistics 2018: GLOBOCAN estimates of incidence and mortality worldwide for 36 cancers in 185 countries. CA Cancer $J$ Clin. 2018;68(6):394-424. doi:10.3322/caac.21492

2. Carnio S, Novello S, Mele T, Levra MG, Scagliotti GV. Extending survival of stage IV non-small cell lung cancer. Semin Oncol. 2014;41(1):69-92. doi:10.1053/j.seminoncol.2013.12.013

3. Cuyun Carter G, Barrett AM, Kaye JA, Liepa AM, Winfree KB, John WJ. A comprehensive review of nongenetic prognostic and predictive factors influencing the heterogeneity of outcomes in advanced non-small-cell lung cancer. Cancer Manag Res. 2014;6:437-449. doi:10.2147/CMAR.S63603

4. Jung CP, Motwani MV, Schwartz GK. Flavopiridol increases sensitization to gemcitabine in human gastrointestinal cancer cell lines and correlates with down-regulation of ribonucleotide reductase M2 subunit. Clin Cancer Res. 2001;7(8):2527-2536.

5. Wong P-P, Bodrug N, Hodivala-Dilke KM. Exploring novel methods for modulating tumor blood vessels in cancer treatment. Curr Biol. 2016;26(21):R1161-R1166. doi:10.1016/j.cub.2016.09.043

6. Folkman J. Angiogenesis inhibitors generated by tumors. Mol Med. 1995;1(2):120-122.

7. Chung AS, Lee J, Ferrara N. Targeting the tumour vasculature: insights from physiological angiogenesis. Nat Rev Cancer. 2010;10 (7):505-514. doi: $10.1038 / \mathrm{nrc} 2868$

8. Abdollahi A, Hahnfeldt P, Maercker C, et al. Endostatin's antiangiogenic signaling network. Mol Cell. 2004;13(5):649-663.

9. O'Reilly MS, Boehm T, Shing Y, et al. Endostatin: an endogenous inhibitor of angiogenesis and tumor growth. Cell. 1997;88 (2):277-285.

10. Kim YM, Jang JW, Lee OH, et al. Endostatin inhibits endothelial and tumor cellular invasion by blocking the activation and catalytic activity of matrix metalloproteinase. Cancer Res. 2000;60 (19):5410-5413.

11. Hanai J, Dhanabal M, Karumanchi SA, et al. Endostatin causes G1 arrest of endothelial cells through inhibition of cyclin D1. $J$ Biol Chem. 2002;277(19):16464-16469. doi:10.1074/jbc.M112274200

12. Folkman J. Antiangiogenesis in cancer therapy-endostatin and its mechanisms of action. Exp Cell Res. 2006;312(5):594-607. doi:10.1016/j.yexcr.2005.11.015

13. Gerlowski LE, Jain RK. Microvascular permeability of normal and neoplastic tissues. Microvasc Res. 1986;31(3):288-305.

14. Dvorak HF, Nagy JA, Dvorak JT, Dvorak AM. Identification and characterization of the blood vessels of solid tumors that are leaky to circulating macromolecules. Am J Pathol. 1988;133(1):95-109.

15. Tong RT, Boucher Y, Kozin SV, Winkler F, Hicklin DJ, Jain RK. Vascular normalization by vascular endothelial growth factor receptor 2 blockade induces a pressure gradient across the vasculature and improves drug penetration in tumors. Cancer Res. 2004;64 (11):3731-3736. doi:10.1158/0008-5472.CAN-04-0074

16. Fukumura D, Jain RK. Tumor microvasculature and microenvironment: targets for anti-angiogenesis and normalization. Microvasc Res. 2007;74(2-3):72-84. doi:10.1016/j.mvr.2007.05.003

17. Jain RK, Duda DG, Clark JW, Loeffler JS. Lessons from phase III clinical trials on anti-VEGF therapy for cancer. Nat Clin Pract Oncol. 2006;3(1):24-40. doi:10.1038/ncponc0403

18. Abraham D, Abri S, Hofmann M, Holtl W, Aharinejad S. Low dose carboplatin combined with angiostatic agents prevents metastasis in human testicular germ cell tumor xenografts. J Urol. 2003;170(4 Pt 1):1388-1393. doi:10.1097/01.ju.0000075917.98929.57
19. Zhu H, Yang X, Ding Y, et al. Recombinant human endostatin enhances the radioresponse in esophageal squamous cell carcinoma by normalizing tumor vasculature and reducing hypoxia. Sci Rep. 2015;5:14503. doi:10.1038/srep14503

20. Ren Z, Wang Y, Jiang W, Dai W, Jiang Y. Anti-tumor effect of a novel soluble recombinant human endostatin: administered as a single agent or in combination with chemotherapy agents in mouse tumor models. PLoS One. 2014;9(9):e107823. doi:10.1371/ journal.pone. 0107823

21. Peng X-C, Qiu M, Wei M, et al. Different combination schedules of gemcitabine with endostar affect antitumor efficacy. Cancer Chemother Pharmacol. 2012;69(1):239-246. doi:10.1007/s00280011-1695-8

22. Eberhard A, Kahlert S, Goede V, Hemmerlein B, Plate KH, Augustin HG. Heterogeneity of angiogenesis and blood vessel maturation in human tumors: implications for antiangiogenic tumor therapies. Cancer Res. 2000;60(5):1388-1393.

23. Giantonio BJ, Catalano PJ, Meropol NJ, et al. Bevacizumab in combination with oxaliplatin, fluorouracil, and leucovorin (FOLFOX4) for previously treated metastatic colorectal cancer: results from the Eastern Cooperative Oncology Group Study E3200. J Clin Oncol. 2007;25 (12):1539-1544. doi:10.1200/JCO.2006.09.6305

24. Ellis LM, Liu W, Ahmad SA, et al. Overview of angiogenesis: biologic implications for antiangiogenic therapy. Semin Oncol. 2001;28(5 Suppl 16):94-104.

25. Okines A, Cunningham D. Current perspective: bevacizumab in colorectal cancer - a time for reappraisal? Eur J Cancer. 2009;45 (14):2452-2461. doi:10.1016/j.ejca.2009.06.028

26. Yang JC, Haworth L, Sherry RM, et al. A randomized trial of bevacizumab, an anti-vascular endothelial growth factor antibody, for metastatic renal cancer. $N$ Engl J Med. 2003;349(5):427-434. doi:10.1056/NEJMoa021491

27. Reck M, Kaiser R, Mellemgaard A, et al. Docetaxel plus nintedanib versus docetaxel plus placebo in patients with previously treated non-small-cell lung cancer (LUME-Lung 1): a phase 3, double-blind, randomised controlled trial. Lancet Oncol. 2014;15 (2):143-155. doi:10.1016/S1470-2045(13)70586-2

28. Zhou S, Yang Y, Yang Y, et al. Combination therapy of VEGF-trap and gemcitabine results in improved anti-tumor efficacy in a mouse lung cancer model. PLoS One. 2013;8(7):e68589. doi:10.1371/journal.pone. 0068589

29. Xu XD, Shao SX, Jiang HP, et al. Warburg effect or reverse Warburg effect? A review of cancer metabolism. Oncol Res Treat. 2015;38 (3): $117-122$. doi: $10.1159 / 000375435$

30. Hwang J-A, Lee EH, Kim H-W, Park JB, Jeon BH, Cho C-H. COMPAng1 potentiates the antitumor activity of 5 -fluorouracil by improving tissue perfusion in murine Lewis lung carcinoma. Mol Cancer Res. 2009;7(12):1920-1927. doi:10.1158/1541-7786.MCR-09-0041

31. Jain RK. Normalization of tumor vasculature: an emerging concept in antiangiogenic therapy. Science. 2005;307(5706):58-62. doi:10.1126/ science. 1104819

32. Armulik A, Genove G, Betsholtz C. Pericytes: developmental, physiological, and pathological perspectives, problems, and promises. Dev Cell. 2011;21(2):193-215. doi:10.1016/j.devcel.2011.07.001

33. Kim KE, Cho C-H, Kim H-Z, Baluk P, McDonald DM, Koh GY. In vivo actions of angiopoietins on quiescent and remodeling blood and lymphatic vessels in mouse airways and skin. Arterioscler Thromb Vasc Biol. 2007;27(3):564-570. doi:10.1161/01.ATV.0000256458.82320.be

34. Matsuo M, Matsumoto S, Mitchell JB, Krishna MC, Camphausen K. Magnetic resonance imaging of the tumor microenvironment in radiotherapy: perfusion, hypoxia, and metabolism. Semin Radiat Oncol. 2014;24(3):210-217. doi:10.1016/j.semradonc.2014.02.002

35. Peng F, Xu Z, Wang J, et al. Recombinant human endostatin normalizes tumor vasculature and enhances radiation response in xenografted human nasopharyngeal carcinoma models. PLoS One. 2012;7 (4):e34646. doi:10.1371/journal.pone.0034646 
36. Huang G, Chen L. Recombinant human endostatin improves anti-tumor efficacy of paclitaxel by normalizing tumor vasculature in Lewis lung carcinoma. J Cancer Res Clin Oncol. 2010;136(8):1201-1211. doi:10.1007/s00432-010-0770-6

37. Lev DC, Ruiz M, Mills L, Mcgary EC, Price JE, Bar-Eli M. Dacarbazine causes transcriptional up-regulation of interleukin 8 and vascular endothelial growth factor in melanoma cells: a possible escape mechanism from chemotherapy. Mol Cancer Ther. 2003;2(8):753-763.

38. Riedel F, Gotte K, Goessler U, Sadick H, Hormann K. Targeting chemotherapy-induced VEGF up-regulation by VEGF antisense oligonucleotides in HNSCC cell lines. Anticancer Res. 2004;24 (4):2179-2183.

39. Sweeney CJ, Miller KD, Sissons SE, et al. The antiangiogenic property of docetaxel is synergistic with a recombinant humanized monoclonal antibody against vascular endothelial growth factor or 2-methoxyestradiol but antagonized by endothelial growth factors. Cancer Res. 2001;61(8):3369-3372.
40. Wang J, Lou P, Lesniewski R, Henkin J. Paclitaxel at ultra low concentrations inhibits angiogenesis without affecting cellular microtubule assembly. Anticancer Drugs. 2003;14(1):13-19.

41. Li N, Zheng D, Wei X, Jin Z, Zhang C, Li K. Effects of recombinant human endostatin and its synergy with cisplatin on circulating endothelial cells and tumor vascular normalization in A549 xenograft murine model. J Cancer Res Clin Oncol. 2012;138(7):1131-1144. doi:10.1007/s00432-012-1189-z

42. Pauwels B, Korst AE, Pattyn GG, et al. Cell cycle effect of gemcitabine and its role in the radiosensitizing mechanism in vitro. Int J Radiat Oncol Biol Phys. 2003;57(4):1075-1083.

43. Xu W-J, Huang C, Wang J, et al. Comparison of the effects of recombinant human endostatin and docetaxel on human umbilical vein endothelial cells in different growth states. Chin Med J. 2011;124(18):2883-2889.

44. Fan J, Du J, Wu J, Fu S, Hu D, Wan Q. Antitumor effects of different administration sequences of cisplatin and Endostar on Lewis lung carcinoma. Oncol Lett. 2015;9(2):822-828. doi:10.3892/ol.2014.2783

\section{Publish your work in this journal}

Cancer Management and Research is an international, peer-reviewed open access journal focusing on cancer research and the optimal use of preventative and integrated treatment interventions to achieve improved outcomes, enhanced survival and quality of life for the cancer patient.
The manuscript management system is completely online and includes a very quick and fair peer-review system, which is all easy to use. Visit http://www.dovepress.com/testimonials.php to read real quotes from published authors. 